\title{
Evidence-based clinical guidelines in Kyrgyz Republic
}

\author{
A.A. Zurdinova* \\ Kyrgyz-Russian Slavic University, Basic and Clinical Pharmacology Department, Bishkek, Kyrgyzstan \\ *Corresponding author. E-mail: aidazur@mail.ru
}

BACKGROUND: Improving quality of care in many countries is one of the priorities of health systems. At the same time one of the most important methods of improving quality of care is the widespread use of methods and principles of evidence-based medicine (EBM) [1]. The implementation of EBM in public health practice provides for the optimization of quality of care in terms of safety, efficacy and cost, one way of which is the use of clinical guidelines. Clinical guidelines developed with the use of EBM, provide an opportunity to use the latest and accurate information to optimize or neutralize impact on physician decision-making of subjective factors such as intuition, expertise, opinion of respected colleagues, recommendations of popular manuals and handbooks, etc.

OBJECTIVE: To assess and analyze the developed clinical guidelines (CG) and protocols (CP) in the Kyrgyz Republic in the period from 2008 to 2014 and evaluate their implementation in practical healthcare.

METHODS: Retrospective analysis of the developed clinical guidelines and protocols according to the approved methodology, interviewing leaders, questioning doctors and patients for their implementation. All participants gave informed consent for voluntary participation in the study.

RESULTS: Within the framework of the National Program "Manas Taalimi" "Strategy for development of evidence-based medicine in the Kyrgyz Republic for 2006-2010” (MOH Order №490 from 09.04.06) was developed and approved for use. Its main purpose was to create a sustainable system of development, deployment and monitoring of the CG and $\mathrm{CP}$ and further promotion of EBM into practical health care, education and science. As a result, a number of documents ("Expert Council for assessing the quality of clinical guidelines/protocols", "AGREE instrument to assess the methodological content of clinical guidelines" [2], "The methodology of development and adaptation of clinical guidelines based on evidence-based medicine") were approved by the Order of the Ministry of Health from 31.12.2008 №704.

This methodology was based on the international guideline SIGN-50 [3], as part of the strategy, it was decided to adapt clinical guidelines of the advanced countries of the world to the organizational characteristics of health care in the Kyrgyz Republic. According to the adopted methodology, the development of clinical guidelines should include the following steps: choose a theme, create a multidisciplinary group to conduct a search of existing clinical guidelines and assess their quality, if necessary, conduct an additional search of evidence, make recommendations and draw up the text of clinical guidelines, conduct peer review and consultations, approve clinical guidelines in the pilot, approve the clinical management of the Ministry of Health, publish and distribute, put into practice, monitor the effectiveness of implementation, provide for the revision and updating of clinical guidelines as new credible information appears. In the future, these CGs will be considered as a basis for the 
development of the $\mathrm{CP}$ in accordance with the possibilities of health care organizations of the country. Figuratively speaking, the CG answers the question - "What can be done in an ideal situation? ", And CP -"What should be done in a country?".

The Ministry of Health over the period 2008-2014 years approved 41 CGs and 118 CPs for common diseases. It should be noted that only $31.7 \%$ of them were represented by the corresponding CGs. Among the approved CPs only $15.3 \%$ were based on the corresponding CGs. All of the CGs and CPs (100\%) identified experts who prepared the documents and to whom they are addressed. The search strategy information was available only in $24.3 \%$ of cases, and only $18.1 \%$ used the criteria for selection of international guidelines, which were found in the CGs. $100 \%$ of the CGs and CPs indicated no conflict of interest of their developers, but it should be noted that $89 \%$ of the CG and CP were developed with the financial assistance of donor organizations supporting the Kyrgyz health reform. The degree of evidence of the recommendations was presented in $100 \%$ of the documents, but grading scales were different: in one CG manual grading was used with 3 levels of evidence (A, B, C), in the other - 4 levels (A, B, C, D ), and in the third - tier 5-6 (I, II, III, IV, V), which is not the approved methodology, which was based on gradation - A, B, C, D. In the process of approval of CGs and CPs, $100 \%$ did not specify points of methodological quality evaluation.

To assess the implementation of approved CG in the practice (training, availability of the CG and CP for each doctor, informing patients about the $\mathrm{CG}$ and $\mathrm{CP}$, monitoring use) we interviewed the leaders of health care organizations (20), surveyed 200 doctors and 100 patients. Only $10 \%$ of leaders said that they participated in the training on the CG and CP. 5\% of them confirmed that every doctor had the corresponding copies of CGs and CPs, $100 \%$ of the leaders conduct internal audits on the use of the CG and CP, in $95 \%$ of cases the developed CGs and CPs do not take into account local health systems conditions (drugs, equipments etc.). $100 \%$ of respondents followed the CGs and CPs, as penalties were introduces by the Ministry of Health, Health Insurance Fund for violation of these recommendations. $25 \%$ of respondents reported improved clinical outcomes. To the question "How to improve the practice of medicine with the use of CGs and CPs?" $100 \%$ of the managers answered that they needed trainings: trainings for physicians, trainings for the developers of these documents. The survey of doctors showed that only $5 \%$ of them were trained in the use of CGs and CPs, $100 \%$ of them had the copies of CGs and CPs, $100 \%$ of doctors answered that the CGs and CPs not always were suitable for their practice. Questioning patients revealed the following: $100 \%$ of them never heard of the CGs and CPs, $2 \%$ of patients noted some improvement in healthcare delivery, and $20 \%$ of patients were referred to private laboratories for diagnostic tests, and $100 \%$ of the patients-respondents bought their drugs for their own pocket money.

CONCLUSIONS: It is very important to ensure equal opportunities in access to medical interventions designed accordingly to the CGs and CPs at all health facilities that will prevent discrimination, depending on territorial distribution, administrative subordination, and other factors in the provision of health care. Implementation of CG and CP recommendations depends not only on the level of health care, knowledge and judgment of a clinician, but also on affordability of a particular diagnostic or therapeutic technologies for a patient. Cases when effective services are not unaffordable for patients should be considered from ethical perspective.

Keywords: Evidence-base guidelines, Kyrgyz Republic, clinical guidelines, clinical protocols, interviewing, survey

\section{References}

[1] VV Vlasov The modern process of standardization in the Russian medicine // Int. Journal of Medical Practice. - 2000. №2. - P. 5-12.1.

[2] AGREE Instrument. The Agree Collaboration. [Internet] 2003 [cited 2003]. Available from: www.agreecollaboration.org.

[3] SIGN 50: A guideline developer's handbook. Scottish Intercollegiate Guidelines Network. - 2001. 